\title{
Modelling of the radiative properties of an opaque porous ceramic layer
}

\author{
Benoit Rousseau • Hector Gomart • \\ Domingos De Sousa Meneses • Patrick Echegut • \\ Mathilde Rieu • Romain Dugas • Pascal Lenormand • \\ Florence Ansart
}

Received: 25 September 2008 / Accepted: 22 December 2009 /Published online: 21 January 2010

(C) The Author(s) 2010. This article is published with open access at Springerlink.com

\begin{abstract}
Solid Oxide Fuel Cells (SOFCs) operate at temperatures above $1,100 \mathrm{~K}$ where radiation effects can be significant. Therefore, an accurate thermal model of an SOFC requires the inclusion of the contribution of thermal radiation. This implies that the thermal radiative properties of the oxide ceramics used in the design of SOFCs must be known. However, little information can be found in the literature concerning their operating temperatures. On the other hand, several types of ceramics with different chemical compositions and microstructures for designing efficient cells are now being tested. This is a situation where the use of a numerical tool making possible the prediction of the thermal radiative properties of SOFC materials, whatever their chemical composition and microstructure are, may be a decisive help. Using this method, first attempts to predict the radiative properties of a lanthanum nickelate porous layer deposited onto an yttria stabilized zirconium substrate can be reported.
\end{abstract}

B. Rousseau $(\varangle) \cdot$ H. Gomart $\cdot$ D. De Sousa Meneses $\cdot$ P. Echegut CEMHTI UPR 3079 CNRS,

$1 \mathrm{D}$ avenue de la Recherche Scientifique,

Orléans, Cedex 02 45071, France

e-mail: benoit.rousseau@cnrs-orleans.fr

B. Rousseau $\cdot$ H. Gomart $\cdot$ D. De Sousa Meneses $\cdot$ P. Echegut

Université d'Orléans, [Polytech Orléans],

Avenue du Parc Floral, BP 6749, Orléans,

Cedex 02 45067, France

M. Rieu $\cdot$ R. Dugas $\cdot$ P. Lenormand $\cdot$ F. Ansart

Institut Carnot CIRIMAT -UMR 5085,

Bât II R1 118, route de Narbonne,

Toulouse, Cedex 09 31062, France

Present Address:

H. Gomart

Laboratoire EM2C CNRS UPR288, Ecole Centrale Paris,

Grande Voie des Vignes,

92295 Châtenay-Malabry, Cedex, France
Keywords Porous ceramic - Thermal radiative properties . Modelling $\cdot$ Infrared spectroscopy

\section{Introduction}

SOFCs are promising power sources that are very attractive due to their high energetic efficiency and low emission of pollutants from the exhaust gases into the environment among others. However, technical bolts, mainly due to their high operating temperature $(\mathrm{T} \sim 1,200 \mathrm{~K})$, impede their industrial development at a reasonable cost. Indeed thermomechanical failures that are observed after thermal cycling reduce the cells' power density and, hence, their reliability. Therefore it is of major importance to predict accurately the temperature field within the cell in order to detect thermal gradients. However, most of the existing thermal models provide contradictory results: some of them clearly ignore the effect of the thermal radiation whereas this mode of energy transfer is important when temperatures are higher than $800 \mathrm{~K}$ [1]. Some authors doubt, the real influence of the thermal radiation on the energy balance models [2]. Moreover, a brief survey of the literature reveals that the thermal radiative properties of the materials used in the design of the core of a SOFC are rare at their operating temperatures. On the other hand, several types of ceramic material are now being studied in order to develop efficient cells. Their shape, microstructure and chemical composition are currently optimized. This picture indicates that the prediction of the radiative properties of these materials require to take into account their internal structure from the atomic scale to the macroscopic scale. This appears to be a promising way to obtain a reliable thermal data bank.

In the present study, a numerical methodology aimed at predicting the thermal radiative of the materials used in the 
design of the cell with a planar geometry is reported. It is based on a Monte Carlo Ray Tracing code. This work is focused on a thick film cathode composed of lanthanum nickelate $\left(\mathrm{La}_{2-\mathrm{x}} \mathrm{NiO}_{4+\delta}\right)$ that is deposited onto yttria stabilized zirconia (YSZ) substrates. The paper is organized as follows: in the first part, the chemical, optical and microstructural behaviours of the ceramic will be described. This information will be used to define in detail the implementation of the Monte Carlo Ray Tracing procedure in the second part. In the third part, the validity of the numerical approach is discussed.

\section{Material characterization}

The method used for the deposition of the $\mathrm{La}_{2} \mathrm{NiO}_{4+\delta}$ layer on the YSZ substrate is briefly described here. Prior to the shaping step of the cathode, the starting material, $\mathrm{La}_{2} \mathrm{NiO}_{4+\delta}$, is prepared in the form of fine powders by a modified Pechini process [3]. During the powder elaboration, the final annealing temperature is selected in order to control the morphology, size, oxygen non-stoechiometry $(\delta)$ and the sintering degree of the oxide powders. The second step of the process consists of dispersing the crystallized powders into an organic mixture with an adequate viscosity [4-6]. The slurry is then deposited onto the YSZ substrate thanks to a dip-coating where the withdrawal is maintained at a controlled speed $(3 \mathrm{~cm} / \mathrm{min})$. After thermal treatment $\left(1150^{\circ} \mathrm{C}, 2 \mathrm{~h}\right)$, the film is continuous, homogeneous, crackfree and adheres well to the YSZ electrolyte substrate.

$\mathrm{X}$-ray diffraction measurements show that the thermal treatment does not induce the appearance of a secondary phase since only the $\mathrm{YSZ}$ and $\mathrm{La}_{2} \mathrm{NiO}_{4+\delta}$ phases are detected. The cathodic layer is composed of grains with a random orientation and a single crystalline phase as is shown by the ESEM (Environmental Scanning Electronic Microscope) image of the cathodic layer (see Fig. 1). After thermal treatment, the size of the grains is approximately $500 \mathrm{~nm}$ and

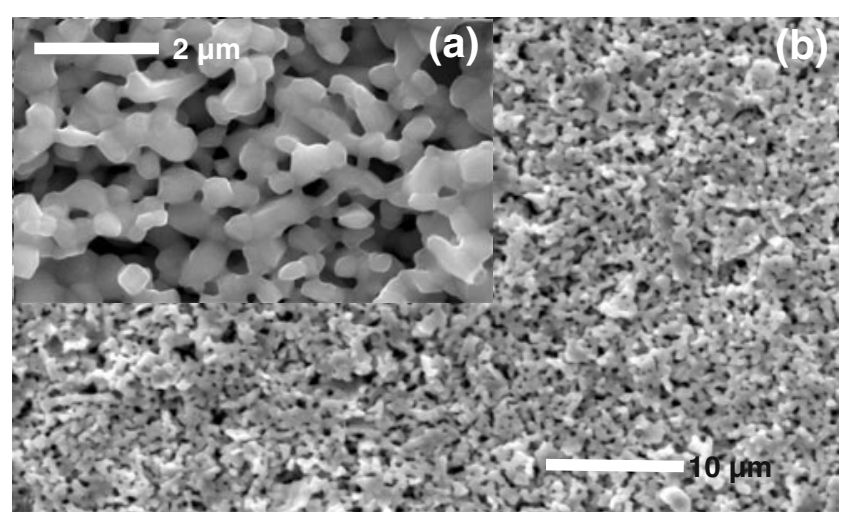

Fig. 1 ESEM image of the cathodic layer. (a) Image obtained with a magnification of 15,000 . (b) Image obtained with a magnification of 2,000 an open connected porosity can be observed. Analysis of the ESEM image roughly indicates that the porosity is $35 \pm 5 \%$.

Note that from an optical viewpoint $\mathrm{La}_{2} \mathrm{NiO}_{4+\delta}$ exhibits a two-dimensional behavior that has been previously recognized by infrared reflectivity on single crystals [7]. The excess oxygen $(+\delta)$ plays a key role in the optical properties of this compound. Indeed, the excess of oxygen create holes directly injected in the two-dimensional layers of these structures. The localization of the electronic carriers, due to their coupling with the lattice, generates a broad absorption band in the mid-infrared range $[8$, 9]. This spectral weight dominates the optical response of a grain in the latter spectral range. Thermogravimetric analysis (TGA) measurements performed on the native powders used for making the slurry indicates that $\delta \sim 0.2$ at $\mathrm{T}=300 \mathrm{~K}$. In this work, it is assumed that the intrinsic optical properties of a small grain can be equivalent to those of a large single crystal of the same chemical composition, but with $\delta=0.14$ [9]. By modeling the infrared reflectivity spectrum acquired in the conducting plane and reported in Ref. [9] with an dielectric function model that makes it possible to reproduce the optical response of mixed ionic-electronic conductors $[8,9]$, the spectral dependence of the dielectric function can be calculated. Then the optical indexes can be easily obtained. The method shows that the value of the extinction coefficient is rather important in the whole spectrum of investigation since its mean value is $30,000 \mathrm{~cm}^{-1}$. This means that the incident light is completely absorbed for a distance superior to $2 \mu \mathrm{m}$ so that the transmission of light through the layer is considered as negligible.

The thickness of the cathodic layer is found to be $8 \mu \mathrm{m}$ by characterizing a cross-section of the film by ESEM so that the layer can be considered as opaque i.e. its normal hemispherical spectral tramittivity is zero. The following material studies will be used as a guideline for developing the strategy of modelling.

\section{Numerical method}

In this work a Monte Carlo Ray Tracing (MCRT) program aimed at reproducing the spectral dependence of thermal radiative properties (reflectivity, transmittivity, emissivity) of heterogeneous material over a large range of temperatures (300 to $1,300 \mathrm{~K}$ ) has been used [10]. Ray Tracing method implies treating the light-matter interaction with the framework of geometric optic approximation [10]. As we have noted previously, the surface is found to be opaque. The code allows for retrieving the directional spectral emissivity, $E$, based on the knowledge of its normal hemispherical spectral reflectivity $R$, since $E=1-R$ according to the Kirchhoff's laws. Therefore, the simulation consists in reproducing the experimental conditions allowing the measurement of the directional hemispherical spectral reflectivity. 
Fig. 2 Three-dimensional image of the cathodic layer. The original image is obtained with a magnification of 4,000. (a) View of the reconstructed image (b) Triangular mesh issued from the reconstructed image (a)

(b)

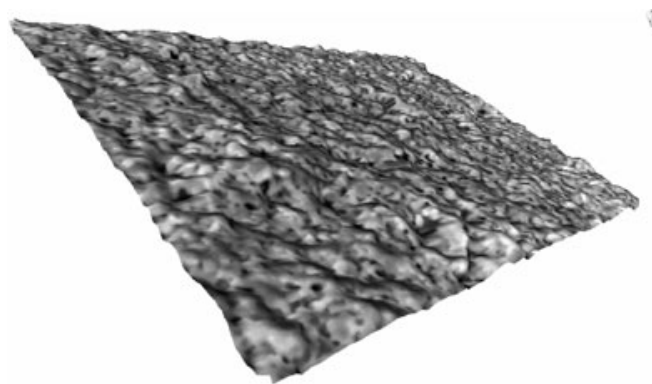

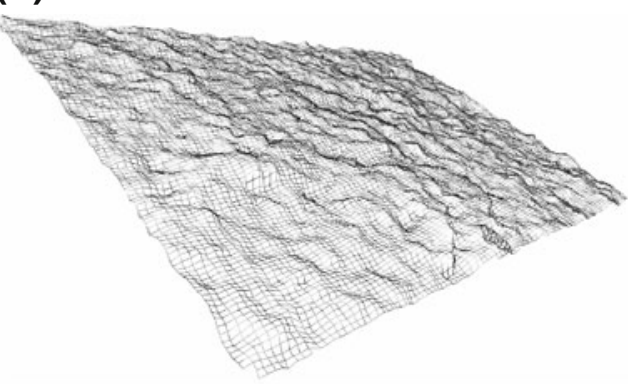

Afterwards an incident beam of $N_{\text {pho }}$ rays, parallel and unpolarized, is launched normally towards the three dimensional reconstructed surface (see Fig. 2). Each ray carries an intensity $I_{0}$ equal to unity. The $x_{0}$ and $y_{0}$ coordinates of the origin of each ray are randomly distributed which has a uniform weight inside the beam, which has a $z_{0}$ constant value. When the ray hits the surface, its new direction is calculated using Snell's reflection law. The local directional spectral reflectivity, $\rho(\sigma, \theta, T)$ is calculated at this point of interaction, by using Fresnel's formulas [10]. Here $\theta$ is the incident angle and $T$ the temperature. The new intensity is then incremented by $I_{i+1}=I_{i} \times \rho(\sigma, \theta, T)$. This recursive process occurs for multiple scattering across the meshing triangular surface until the ray leaves the surface or when its intensity is smaller than a fixed value $I_{\min }$, at which point the photon is considered to be absorbed with an intensity equal to zero. Following this operation, another photon is launched. Then a storage procedure counts respectively the numbers of scattered and absorbed photons. The hemispherical spectral reflectivity turns out to be the ratio of the number of scattered photons to the total number of photons.

\section{Results and discussion}

The first step of the modelling consists in obtaining a three dimensional image of the ceramic surface. The ESEM apparatus is equipped with an eucentric stage that allows for the acquisition of tilted ESEM images with a small angle of tilting $\left(0,+6^{\circ}\right)$. The Mex ${ }^{\circledR}$ software is then used to combine both the stereo images in order to calculate the local height, $z(x, y)$, that corresponds to each pixel $(x, y)$ of the ESEM image. It makes possible to reconstruction of the three dimensional image of the observed sample (Fig. 2). The pixel size is $0.179 \mu \mathrm{m}$.

The three-dimensional numerical image of the cathodic layer is injected into the MCRT code. The calculation of the local directional reflectivity $\rho(\sigma, \theta, T)$ that must to be known at each point of interaction, that a ray intercepts a triangle, is based on the optical indexes measured on the $\mathrm{La}_{2} \mathrm{NiO}_{4.14}$ single crystal at room temperature as previously described [8]. The incident beam size is $0.2 \mathrm{~mm}$ and the $N_{p h o}=10^{5}$ photons. The result of this modelling as well as the calculated normal spectral emissivity of the single crystal (solid black curve) is shown in Fig. 3 (square symbol). The increase of the emissivity due to the surface roughness in the whole spectral range is in the order of $8 \%$. Figure 3 shows the value of the experimental spectral emissivity (grey solid curves) acquired at $\mathrm{T}=900 \mathrm{~K}$, thanks to an experimental set-up used in Ref. [11, 12].

Even if the temperatures are not the same, this latter curve can be used as a guideline to discuss the validity of the model. It shows that there is a significant discrepancy between the experimental data and the simulated one.

To go one step further, the use of the Effective Medium Approximation (EMA) allows for taking into account the internal porosity of the layer [13]. The EMA is based on the fact that a radiation of a given wavelength $\gamma \lambda$, propagates in a heterogeneous medium as if it were homogeneous. It requires that the pore/grain length scale be much smaller than the incident wavelength that exists in this work (pore size is centered on $400 \mathrm{~nm}$ (Fig. 1a)). Hence, the propagation of radiation can be studied, in a much simpler way, by introducing a homogeneous equivalent effective dielectric function, $\widetilde{\varepsilon}_{\text {eff }}$. The Bruggemann's model has been

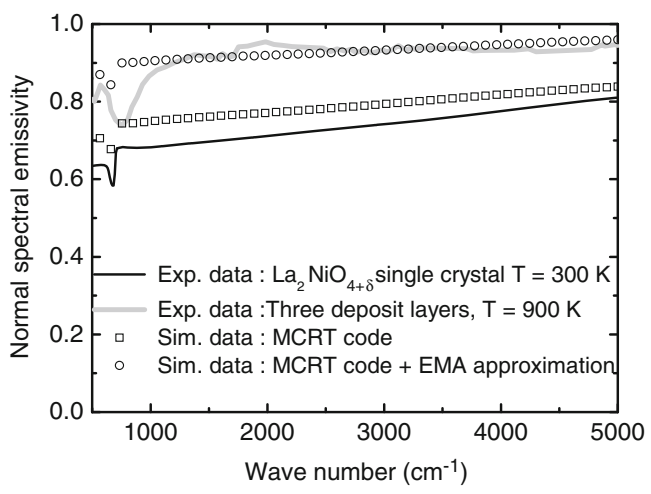

Fig. 3 Comparison of experimental (solid line) and numerical (symbols) normal spectral emissivity for the F3 sample that is composed of three $\mathrm{La}_{2-x} \mathrm{NiO}_{4+\delta}$ layers 
used [14]. The effective dielectric function depends on the porosity, $p$, the dielectric function of $\mathrm{La}_{2} \mathrm{NiO}_{4.14}$, and the one of the air $(\sim 1)$ :

$p \frac{\widetilde{\varepsilon}_{\text {air }}-\widetilde{\varepsilon}_{\text {eff }}}{\widetilde{\varepsilon}_{\text {air }}+2 \widetilde{\varepsilon}_{\text {eff }}}+(1-p) \frac{\widetilde{\varepsilon}_{L a_{2} \mathrm{NiO}_{\mathrm{t}_{4} 14}}-\widetilde{\varepsilon}_{\text {eff }}}{\widetilde{\varepsilon}_{\mathrm{La}_{2} \mathrm{NiO}_{4.14}}+2 \widetilde{\varepsilon}_{\text {eff }}}=0$

The application of Eq. 1 with $p \sim 35 \%$ makes it possible to obtain new effective optical index that is injected in the MCRT code. The result of the new simulation is reported in Fig. 3 (circle symbol). Overall, a satisfactory good agreement is achieved for F3. Moreover, the homogenized layer can be considered as opaque. It shows that a combination of two approaches that take account of the chemical composition and the microstructure (surface roughness, porosity, grain/pore size distribution) of the layer can model its optical response on a wide domain of wave numbers.

\section{Conclusion}

This study has investigated the relationship between the normal spectral emissivity of an opaque thick layer of $\mathrm{La}_{2} \mathrm{NiO}_{4+\delta}$ and both its internal microstructure and chemical composition. A strategy of modelling based on a Monte Carlo Ray Tracing code was implemented in order to take account of each contribution from the atomic scale to the macroscopic scale. It shows that the chemical composition, the thickness, the surface roughness, the porosity and the pore size mainly act on the optical response of the coating. Much works are required to model the optical response of the thinner layers. Moreover, on a thermal radiative point of view, it is experimentally shown that for critical thickness the cathode layer can evolve from a semitransparent optical behaviour to an opaque one. It indicates that the methodol- ogy used to treat heat transfer within a SOFC cell at its operating temperature must carefully integrate the size, the chemical composition and the microstructure of the cell materials.

Acknowledgments The authors gratefully acknowledge the contribution of E Veron (CEMHTI, Orléans) for the ESEM characterization. We also acknowledge financial support by ANRJC07_190903 THERMASOFC

Open Access This article is distributed under the terms of the Creative Commons Attribution Noncommercial License which permits any noncommercial use, distribution, and reproduction in any medium, provided the original author(s) and source are credited.

\section{References}

1. S. Murthy, A.G. Fedorov, J. Power Sources 124, 453-458 (2003)

2. K.J. Daun, S.B. Beatle, F. Liu, G.J. Smallwood, J. Power Sources 157, 302-310 (2006)

3. P. Pechini, Patent 3.330.697, 11 July 1967

4. S. Castillo, R.F. Cienfuegos, M.L. Fontaine, P. Lenormand, P. Bacchin, F. Ansart, Mat. Res. Bull 42, 2125-2131 (2007)

5. M. Greenblatt, Z. Zhang, M.H. Whangbo, Synth. Met. 85, 14511452 (1997)

6. T. Chartier, D. Merle, J.L. Besson, J. Eur. Ceram. Soc. 15, 101107 (1995)

7. J.M. Bassat, P. Odier, F. Gervais, Phys. Rev B. 35, 7126 (1987)

8. B. Rousseau, J.M. Bassat, A. Blin, M. Silva de Oliveira, P. Odier, C. Marin, P. Simon, Solid State Sci 6, 1131-1137 (2004)

9. B. Rousseau, D. De Sousa Meneses, A. Blin, M. Chabin, P. Echegut, P. Odier, F. Gervais, Phys. Rev. B 72, 104114 (2005)

10. B. Rousseau, D. De Sousa Meneses, P. Echegut, M. Di Michiel, J.-F. Thovert, Appl Opt 46, 4266-4276 (2007)

11. O. Rozenbaum, D. De Sousa Meneses, S. Chermanne, Y. Auger, P. Echegut, Rev. Sci. Instrum. 70, 4020-4025 (1999)

12. B. Rousseau, J.-F. Brun, D. De Sousa Meneses, P. Echegut, Int. J. Thermophysic 26, 1277-1285 (2005)

13. D.A. Aspnes, Thin Solid Films 89, 249-262 (1982)

14. D.A.G. Bruggeman, Ann. Phys. (Leipzig) 24, 636 (1935) 